DOI: $10.15593 / 2224-9982 / 2019.59 .04$

УДК 621.791.94.034

\author{
Ю.В. Цветков ${ }^{1}$, Г.А. Цветков ${ }^{2,3}$, Р.В. Цветков ${ }^{4}$, \\ В.И. Малинин ${ }^{2}$, М.А. Савин ${ }^{2}$, Н.Е. Жижилев ${ }^{5}$ \\ ${ }^{1}$ Малое инновационное предприятие «Энергомашиностроение», Пермь, Россия \\ ${ }^{2}$ Пермский национальный исследовательский политехнический университет, Пермь, Россия \\ ${ }^{3}$ Пермский государственный научный исследовательский университет, Пермь, Россия \\ ${ }^{4}$ Лаборатория интеллектуального мониторинга ИМСС УрО РАН, Пермь, Россия \\ ${ }^{5}$ Публичное акционерное общество «Протон - Пермские моторы», Пермь, Россия

\section{РЕЗУЛЬТАТЫ ЭКСПЕРИМЕНТАЛЬНОГО ИССЛЕДОВАНИЯ ПРОЦЕССОВ ГОРЕНИЯ КИСЛОРОДНОГО КОПЬЯ И ОПРЕДЕЛЕНИЕ ЗАВИСИМОСТИ ОСНОВНЫХ ВЫХОДНЫХ ХАРАКТЕРИСТИК ОТ ВХОДНЫХ ПАРАМЕТРОВ}

\begin{abstract}
Результатом работы является комплекс количественных и качественных характеристик процессов горения кислородного копья, а также процессов его взаимодействия с разрезаемым материалом различного состава.

Установлены зависимости основных выходных характеристик от входных параметров.

Комплекс полученных характеристик включает в себя, в частности, абсолютные значения температуры горения топливных композиций на основе железа с алюминием в качестве горючего и газообразного кислорода в качестве окислителя соответственно. Таким образом, при различных сочетаниях упомянутых компонентов полученные значения температуры огневого пучка составили широкий диапазон: от 1800 до 4050 К с соответствующим химическим составом продуктов сгорания всех топливных композиций и агрегатных состояний этих продуктов.

Были выполнены гидродинамические расчеты процесса течения газообразного кислорода в полости кислородного копья с получением, в частности, численных значений скорости и расхода кислорода, а также полей их распределения в полости копья и на выходе из него в различные стадии горения.

Проведенные расчетные, проектные и экспериментальные исследования обеспечивают обоснованный выбор оптимальной конструктивной схемы и параметров рабочего процесса кислородного копья для его работы в составе аппаратного комплекса при решении многопараметрических задач по разделительной и иной резке изделий в различных условиях: в производственных условиях; в условиях ликвидации аварий, последствий чрезвычайных ситуаций и проведении антитеррористических операций.

Ключевые слова: кислородное копье, алюминиевая проволока, кислородная резка, увеличение температуры горения копья, скорость резания кислородным копьем.
\end{abstract}

\author{
Yu.V. Tsvetkov ${ }^{1}$, G.A. Tsvetkov ${ }^{2,3}$, R.V. Tsvetkov ${ }^{4}$, \\ V.I. Malinin', M.A. Savin'², N.E. Zhizhilev ${ }^{5}$ \\ ${ }^{1}$ Small Innovative Enterprise "Energomashinostroenie", Perm, Russian Federation \\ ${ }^{2}$ Perm National Research Polytechnic University, Perm, Russian Federation \\ ${ }^{3}$ Perm State National Research University, Perm, Russian Federation \\ ${ }^{4}$ Laboratory of Intellectual Monitoring, Institute of Continuous Media Mechanics \\ of the Ural Branch of the Russian Academy of Science, Perm, Russian Federation \\ ${ }^{5}$ Public Joint Stock Company "Proton - Permskiye motory", Perm, Russian Federation
}

\title{
THE RESULTS OF EXPERIMENTAL STUDIES OF COMBUSTION PROCESSES \\ THE OXYGEN LANCE AND THE ESTIMATION OF THE MAIN OUTPUT PARAMETERS FROM INPUT PARAMETERS
}

The results of the work are a complex of quantitative and qualitative characteristics of the combustion processes of an oxygen spear, as well as the processes of its interaction with cut material of various compositions.

The dependences of the main output characteristics on the input parameters are established.

The set of characteristics obtained includes, in particular, the absolute values of the combustion temperatures of ironaluminum-based fuel compositions as combustible and gaseous oxygen as an oxidizing agent, respectively. Thus, with various combinations of the mentioned components, the obtained values of the temperatures of the firing beam made up a wide range: 
from $\sim 1800 \mathrm{~K}$ to $\sim 4050 \mathrm{~K}$ with the corresponding chemical compositions of the combustion products of all fuel compositions and the aggregate states of these products.

Hydrodynamic calculations of the process of the flow of gaseous oxygen in the cavity of the oxygen spear were also performed, in particular, the numerical values of the velocities and consumption of oxygen, as well as the fields of their distribution in the cavity of the spear and at the exit from it to various stages of combustion, were obtained.

The carried out calculation, design and experimental studies provide a reasonable choice of the optimal design scheme and parameters of the oxygen spear working process for its operation as part of the hardware complex when solving multiparameter problems in dividing and other cutting of products in various conditions: in production conditions; in the conditions of liquidation of accidents, consequences of an emergency and anti-terrorist operations.

Keywords: oxygen lance, aluminum wire, flame cutting, an increase in the temperature of the burning spear, cutting speed oxygen lance.

\section{Введение}

Среди основных этапов исследования необходимо выделить разработку кислородного копья и численное моделирование процесса резки [1-4].

Сложность задачи объясняется множеством процессов, протекающих в ходе резки, во время которой происходит горение топливных композиций, а также материала трубки на конце кислородного копья.

При моделировании кислородного копья ставится серьезная задача моделирования течения окислителя в тракте копья [5]. Сложность заключается в форме каналов, образующихся между проволоками в результате сборки кислородного копья, а также в наличии сверхзвукового течения на конце копья в процессе его горения.

В зависимости от различного соотношения горючего и окислителя становится возможным решение некоторых поставленных задач.

Нужно сказать, что для проведения процесса резки требуется наличие качественной исходной информации в связи с наличием фазового перехода в разрезаемом материале. Для получения достоверных данных все расчеты проведены в специализированной программе Astra.

\section{Исследование процесса горения кислородного копья и определение зависимости основных выходных характеристик от входных параметров}

Исследование проводится с целью получения зависимости выходных характеристик процесса горения (температура, состав продуктов сгорания, теплофизические характеристики и т.п.) материала кислородного копья от входных параметров (исходный химический состав материалов и их соотношение).

В основу положена методика выполнения термодинамических расчетов по одной из ранее созданных в этой области программ (Astra, «Плазма») с получением необходимых характеристик и зависимостей, а также последующим анализом полученных результатов применительно к процессу горения кислородного копья.

Материалами, формирующими «тело» кислородного копья, являются:

- низкоуглеродистая сталь (Ст10 или Ст20), принимаемая в нашем расчете как чистое железо без существенных потерь точности;

- алюминий, который принимаем без примесей с меньшими издержками по точности расчетов.

Конструктивно кислородное копье представляет собой стальную трубку, в которой уложены алюминиевые либо железные проволоки. Проволоки в трубке могут находиться как наиболее плотно с учетом допусков, так и произвольным образом, в зависимости от их диаметра и требуемого расхода окислителя $[6,7]$.

Исследуемыми топливными композициями (горючее + окислитель) в произведенных расчетах являются следующие:

1. $\mathrm{Al}+\mathrm{O}_{2}$.

2. $\mathrm{Fe}+\mathrm{O}_{2}$.

3. $(\mathrm{Al}+\mathrm{Fe})+\mathrm{O}_{2}$. 
Все исследуемые топливные композиции приведены в шести различных соотношениях по массовым долям.

Условия процесса горения кислородного копья следующие [8]:

- давление атмосферное 0,1 МПа;

- массовое соотношение компонентов (окислитель/горючее), принимаемое $\kappa=0,5 ; 0,75$; 1,$0 ; 1,5 ; 2,0 ; 3,0$;

- распределение расхода компонентов по фронту горения (по торцу кислородного копья) равномерное, а «смесеобразование» принимаем идеальным.

В результате расчета получены все заданные выходные характеристики процесса горения кислородного копья.

Изучив данные проведенных расчетов, построим зависимости, иллюстрирующие максимальную температуру горения при различном соотношении окислителя/горючего.

При использовании алюминия в качестве горючего в соотношении с кислородом, равном 1 (50 \% кислорода, 50 \% алюминия), достигается максимальная температура горения 4029 K (рис. 1).

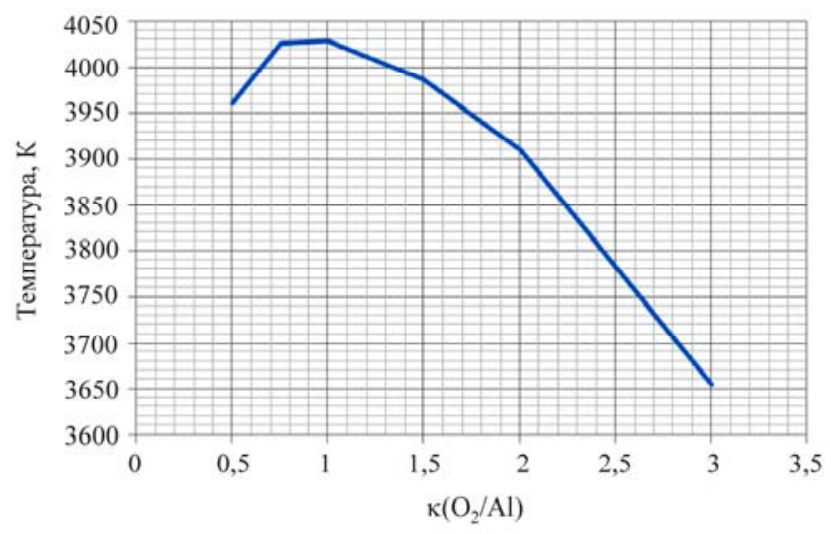

\begin{tabular}{|c|c|}
\hline \multicolumn{2}{|c|}{$\mathrm{O}_{2} / \mathrm{Al}$} \\
\hline kappa & $T, \mathrm{~K}$ \\
\hline 0,499925 & 3960,4 \\
\hline 0,751313 & 4026,4 \\
\hline 1 & 4029 \\
\hline 1,5 & 3987,1 \\
\hline 2,0003 & 3910,2 \\
\hline 3 & 3654,4 \\
\hline
\end{tabular}

Рис. 1. Распределение значений температуры горения для алюминия (Al)

При использовании в качестве горючего железа ситуация меняется. Для железа наилучшее соотношение, обеспечивающее максимальную температуру горения $3118,4 \mathrm{~K}$, равно 0,25 (20\% кислорода, $80 \%$ железа) (рис. 2).

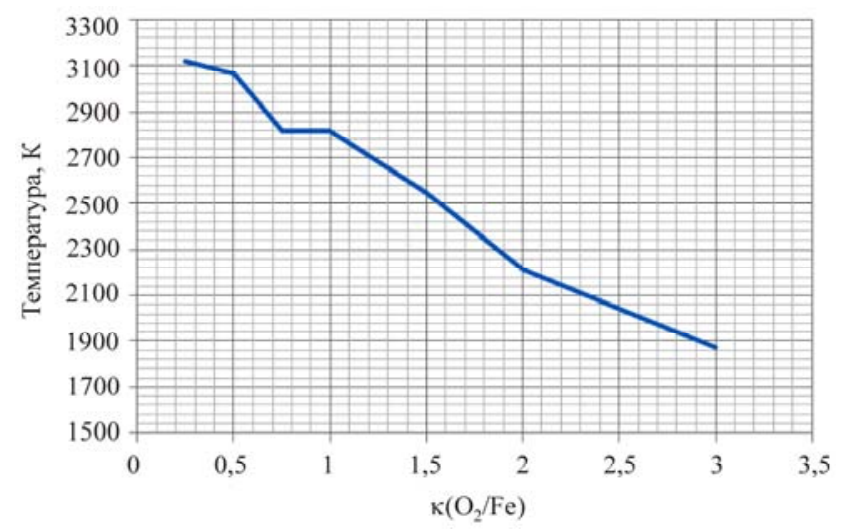

\begin{tabular}{|c|c|}
\hline \multicolumn{2}{|c|}{$\mathrm{O}_{2} / \mathrm{Fe}$} \\
\hline kappa & $T, \mathrm{~K}$ \\
\hline 3 & 1869,7 \\
\hline 2,0003 & 2211,9 \\
\hline 1,5 & 2546,6 \\
\hline 1 & 2816,7 \\
\hline 0,751313 & 2816,7 \\
\hline 0,499925 & 3066,2 \\
\hline 0,25 & 3118,4 \\
\hline
\end{tabular}

Рис. 2. Распределение температуры горения для железа (Fe)

Похожая зависимость наблюдается при использовании смеси железа и алюминия в качестве горючего. В этом варианте максимальную температуру 3364,5 К обеспечивает следующее соотношение окислителя/горючего: 33,33 \% кислорода, 16,67 \% алюминия, 50 \% железа. Результаты представлены на рис. 3 . 


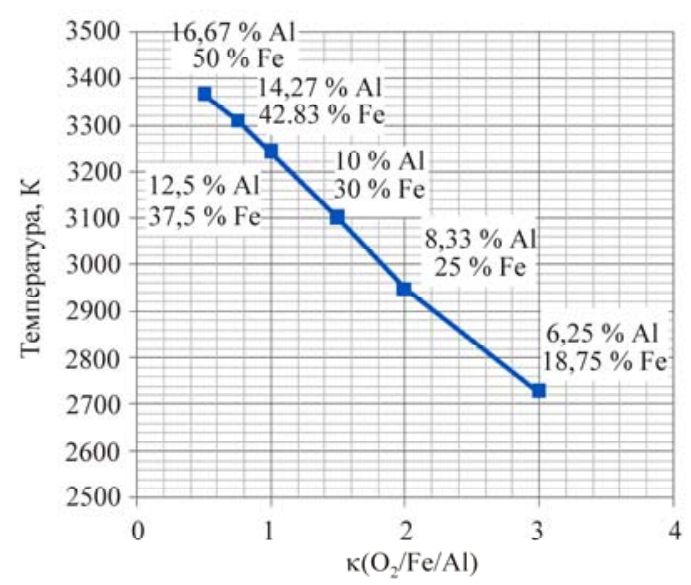

\begin{tabular}{|c|c|c|c|}
\hline \multicolumn{4}{|c|}{$\mathrm{O}_{2} / \mathrm{Fe} / \mathrm{Al}$} \\
\hline kappa & $T, \mathrm{~K}$ & $\mathrm{Al}, \%$ & $\mathrm{Fe}, \%$ \\
\hline 3 & 2728,2 & 6,25 & 18,75 \\
\hline 2,0003 & 2948 & 8,33 & 25 \\
\hline 1,5 & 3099,2 & 10 & 30 \\
\hline 1 & 3241,4 & 12,5 & 37,5 \\
\hline 0,751313 & 3309,4 & 14,27 & 42,83 \\
\hline 0,499925 & 3364,5 & 16,67 & 50 \\
\hline
\end{tabular}

Рис. 3. Распределение температуры для смеси $\left(\mathrm{O}_{2} / \mathrm{Fe} / \mathrm{Al}\right)$

Рассмотрим также изменение массовых долей некоторых веществ, входящих как в продукты сгорания, так и в горючее, в зависимости от соотношения окислителя/горючего (рис. 4-6).

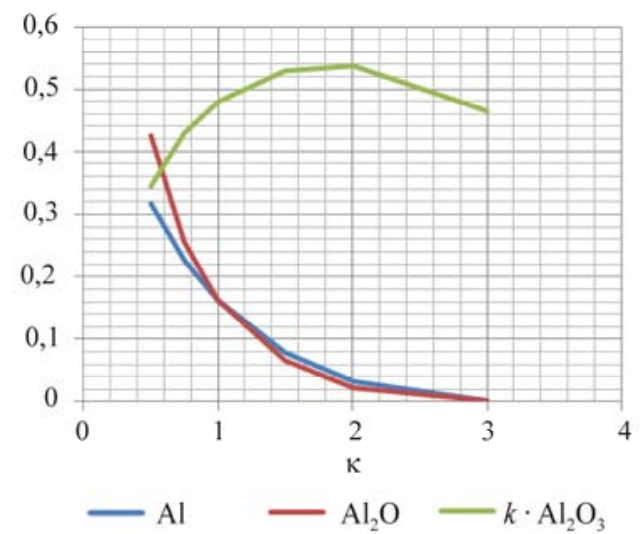

\begin{tabular}{|c|c|c|c|}
\hline \multicolumn{4}{|c|}{$\mathrm{O}_{2} / \mathrm{Al}$} \\
\hline kappa & $\mathrm{Al}$ & $\mathrm{Al}_{2} \mathrm{O}$ & $k \cdot \mathrm{Al}_{2} \mathrm{O}_{3}$ \\
\hline 0,499925 & 0,31844 & 0,42577 & 0,34399 \\
\hline 0,751313 & 0,22676 & 0,25559 & 0,42952 \\
\hline 1 & 0,16145 & 0,16213 & 0,47972 \\
\hline 1,5 & 0,079121 & 0,064964 & 0,52994 \\
\hline 2,0003 & 0,033111 & 0,02177 & 0,53847 \\
\hline 3 & 0,002323 & 0,000801 & 0,46612 \\
\hline
\end{tabular}

Рис. 4. Распределение некоторых компонентов при горении алюминия

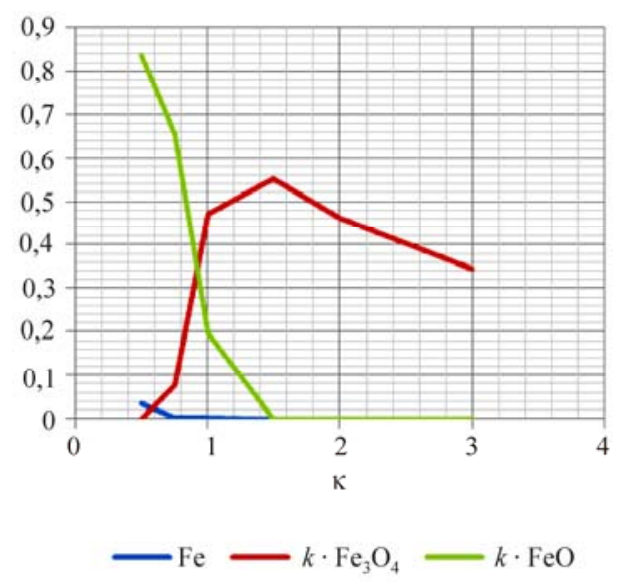

\begin{tabular}{|c|c|c|c|}
\hline \multicolumn{5}{|c|}{$\mathrm{O}_{2} / \mathrm{Fe}$} \\
\hline kappa & $\mathrm{Fe}$ & $k \cdot \mathrm{Fe}_{3} \mathrm{O}_{4}$ & $k \cdot \mathrm{FeO}$ \\
\hline 3 & $1,1025 \mathrm{E}-09$ & $3,46 \mathrm{E}-01$ & 0 \\
\hline 2,0003 & $1,19 \mathrm{E}-06$ & 0,46059 & 0 \\
\hline 1,5 & $1,68 \mathrm{E}-04$ & 0,55206 & 0 \\
\hline 1 & $3,85 \mathrm{E}-03$ & 0,47037 & 0,19868 \\
\hline 0,751313 & $3,85 \mathrm{E}-03$ & 0,078041 & 0,65656 \\
\hline 0,499925 & 0,033023 & 0 & 0,83719 \\
\hline
\end{tabular}

Рис. 5. Распределение некоторых компонентов при горении железа 


\begin{tabular}{|c|c|c|c|c|c|}
\hline \multicolumn{7}{|c|}{$\mathrm{O}_{2} / \mathrm{Fe} / \mathrm{Al}$} \\
\hline kappa & $k \cdot \mathrm{FeO}$ & $\mathrm{Fe}$ & $k \cdot \mathrm{Al}_{2} \mathrm{O}_{3}$ & $\mathrm{Al}$ & $\mathrm{Fe}$ \\
\hline 3 & 0 & $1,48 \mathrm{E}-03$ & 0,11809 & 6,25 & 18,75 \\
\hline 2,0003 & 0,29164 & 0,012382 & 0,15739 & 8,33 & 25 \\
\hline 1,5 & 0,30631 & 0,043045 & 0,18892 & 10 & 30 \\
\hline 1 & 0,29684 & 0,13376 & 0,23611 & 12,5 & 37,5 \\
\hline 0,751313 & 0,27387 & 0,23885 & 0,26949 & 14,27 & 42,83 \\
\hline
\end{tabular}

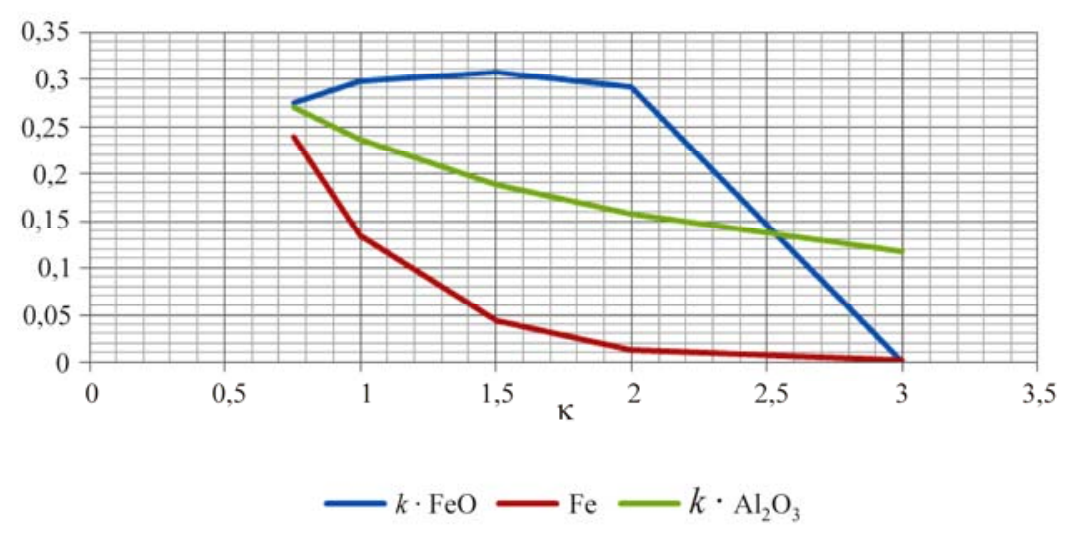

Рис. 6. Распределение некоторых компонентов при горении смеси

При анализе графиков становится видно, что практически во всех случаях максимальная температура горения соответствует максимальной эффективности сгорания горючего. Но для горения железа требуется существенно меньше кислорода, чем для горения алюминия, поэтому использование чистого алюминия в качестве горючего для кислородных копий нецелесообразно.

Таким образом, по результатам можно судить об эффективности использования горючего (полноте его сгорания) и в совокупности с данными, демонстрирующими изменение температуры, можно добиться оптимального соотношения, позволяющего достичь максимальной эффективности копья.

\section{Расчетно-теоретические исследования гидродинамических процессов, протекающих в тракте кислородного копья различных конструктивных схем}

Воспользуемся результатами выполненных термодинамических расчетов при разработке конструктивных схем кислородных копий различного назначения.

Для инициирования и поддержания выбранного для реальной работы режима горения кислородного копья (по температуре огневого пучка, массовому расходу продуктов сгорания и др.) необходимо обеспечить «участие» в процессе горения расчетных масс горючего (железа, алюминия и т.п.) и окислителя (кислорода), а точнее их массовых расходов, в определенном заданном соотношении на протяжении всего процесса горения [9-14].

Для выполнения этих требований необходимо сконструировать кислородное копье так, чтобы после заполнения проволокой полости трубки в ней оставались продольные каналы, суммарная площадь поперечного сечения которых, а также другие гидравлические характеристики (смоченный периметр, шероховатость и т.п.) обеспечивали заданный массовый расход окислителя при постоянном давлении кислорода на входе в кислородное копье.

Это достигается варьированием значений внутреннего диаметра трубки, а также значений диаметра, количества и способов укладки проволоки в трубке. 
В нашем случае для гидродинамических расчетов используем трубку с круглым внутренним поперечным сечением и проволоку круглого сечения.

Гидравлические расчеты проводятся на базе ПО MATLAB, в котором программируется расчетная схема. В соответствии с этой схемой решается задача продувки холодного кислородного копья кислородом при заданных значениях давления на входе и выходе копья.

Гидродинамические расчеты проводятся на кислородных копьях без мундштуков, так как при рабочих значениях давления кислородного копья падение давления на мундштуке несущественно. При выборе максимально возможных диаметров и длины копья $(2,75$ м) скорость течения кислорода равна 31,5 м/с, что соответствует турбулентному режиму $(\operatorname{Re}=24692,11)$. При этом падение давления на этом участке составит всего 333,407 Па при коэффициенте шероховатости

$$
\lambda=\frac{0,3165}{\operatorname{Re}^{0,25}}=0,2525 .
$$

Скорость и объемный расход кислорода рассчитываются по перепаду давления исходя из уравнения Дарси - Вейсбаха:

$$
\Delta P=\frac{\varepsilon \omega^{2} \rho}{2}
$$

где $\varepsilon$ - коэффициент местного сопротивления; $\omega$ - скорость течения потока; $\rho$ - плотность прокачиваемой среды.

Коэффициент местного сопротивления определяется с учетом коэффициента гидравлического сопротивления и характеристик канала:

$$
\varepsilon=\frac{\lambda L}{D_{\text {eqv }}}
$$

где $L$ - длина канала; $D_{\text {eqv }}$ - эквивалентный диаметр.

Для определения коэффициента гидравлического сопротивления требуется число Рейнольдса, поэтому перед началом итерационного процесса значению скорости присваивается некоторое начальное приближение. Расчет же эквивалентного диаметра ведется с учетом периметра смоченной поверхности и живого сечения.

С учетом вышесказанного для расчета требуются следующие уравнения:

$$
\begin{gathered}
\lambda=\frac{0,3165}{\operatorname{Re}^{0,25},} \\
\operatorname{Re}=\frac{\omega D_{\text {eqv }}}{v}, \\
P=\pi(D+19 d),
\end{gathered}
$$

где $P$ - периметр смоченной поверхности; $D$ - внутренний диаметр трубы; $d$ - диаметр проволоки; $v$ - кинематическая вязкость.

Эквивалентный диаметр - отношение живого сечения к периметру смоченной поверхности:

$$
D_{\text {eqv }}=\frac{4 D_{w}}{P} .
$$

Массовый расход пересчитывается с учетом скорости течения по формуле

$$
G_{m}=\rho \omega D_{w} ;
$$




$$
D_{w}=\pi\left(\frac{D^{2}}{4}-\frac{n d^{2}}{4}\right)
$$

По описанной методике требуется провести серию гидравлических расчетов.

Для всех расчетов, где не указано дополнительно, принимаются следующие исходные данные:

Избыточное давление на входе 12 бар.

Избыточное давление на выходе. 0 бар.

Температура кислорода $300 \mathrm{~K}$.

Плотность кислорода $1,30878 \kappa \kappa / \mathrm{M}^{3}$.

Кинематическая вязкость кислорода $1,5696 \mathrm{~m}^{2} / \mathrm{c}$.

Серия расчетов первой группы направлена на анализ течения кислорода в кислородном копье в зависимости от изменения длины копья. Это важный момент, поскольку при работе копья его длина постепенно уменьшается в процессе сгорания.

Для расчетов приняты основные исходные данные (см. выше). При этом внутренний диаметр трубки составляет 12 мм, диаметр проволок 2,4 мм, количество проволок - 19 шт., схема укладки - $(1+6+12)$. Длины кислородного копья, принятые к расчету: 2,$75 ; 1,25 ; 0,25$ м.

По результатам расчетов наблюдается резкое увеличение числа Рейнольдса, скорости течения кислорода и массового расхода с уменьшением длины копья (рис. 7).
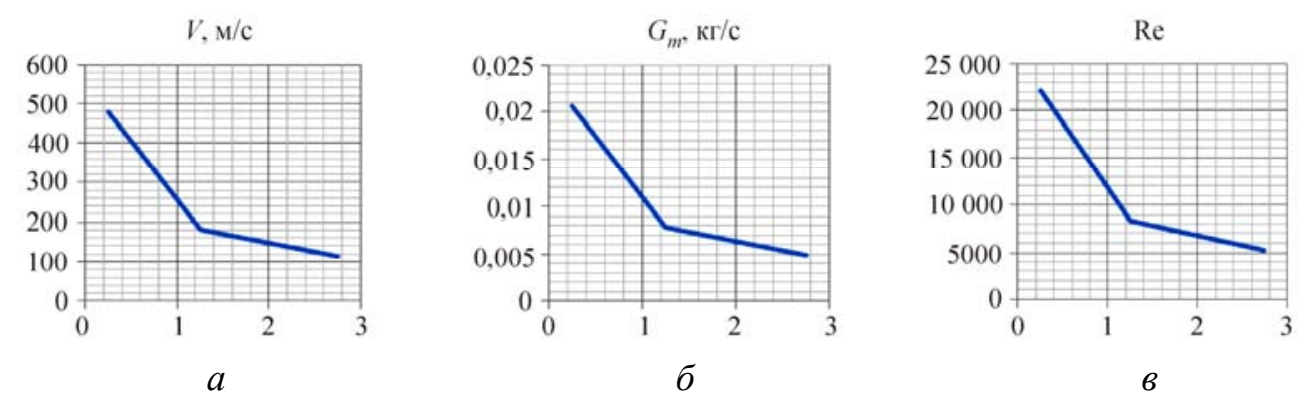

Рис. 7. Зависимости скорости (a), массового расхода (б), числа Рейнольдса (в) от длины копья

Точные значения параметров при различных значениях длины сведены в табл. 1.

Таблица 1

Расчетные параметры

\begin{tabular}{|c|c|c|c|}
\hline Длина, м & Скорость, м/с & Массовый расход, кг/с & $\operatorname{Re}$ \\
\hline 2,75 & 114 & 0,0049 & $5,25 \mathrm{E}+03$ \\
\hline 1,25 & 181 & 0,0078 & $8,33 \mathrm{E}+03$ \\
\hline 0,25 & 480 & 0,0206 & $2,21 \mathrm{E}+04$ \\
\hline
\end{tabular}

При более точном исследовании скорости течения и длины копья можно построить следующую зависимость, представленную на рис. 8.

Для демонстрации нелинейной зависимости была проведена дополнительная серия расчетов, в которой для простоты анализировалась лишь скорость течения.

Увеличение скорости и, следовательно, расхода кислорода возникает при уменьшении длины копья более чем наполовину. При этом ожидается пропорциональное увеличение мощности кислородного копья, скорости резания и снижение затраченного на разрезание материала времени. 


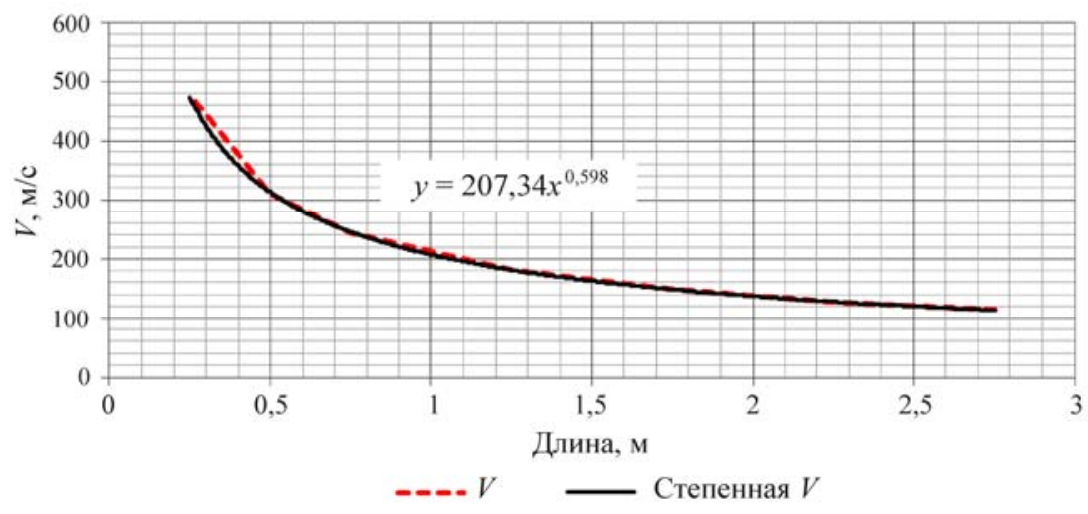

Рис. 8. Зависимость скорости от длины копья

Исходя из расчетных данных можно сделать вывод о необходимом снижении длины кислородного копья при условии выполнения требований безопасности при его эксплуатации и экономической обоснованности.

\section{Влияние технологического разброса параметров}

Проведенный расчет показал, что небольшое изменение, вызванное технологическим разбросом параметров изготавливаемых промышленным способом проволок и трубки, окажет существенное влияние на скорость течения и расход кислорода через сечение кислородного копья.

Для наглядности и информативности все расчетные и дополнительные исходные данные, касающиеся геометрии копья, сведены в табл. 2 и 3.

Таблица 2

Характеристики течения (при одновременном уменьшении значений диаметра проволок и внутреннего диаметра трубы)

\begin{tabular}{|c|c|c|c|c|c|}
\hline Длина, м & $\begin{array}{c}\text { Диаметр } \\
\text { трубы, мм }\end{array}$ & $\begin{array}{c}\text { Диаметр } \\
\text { проволок, мм }\end{array}$ & $\begin{array}{c}\text { Скорость, } \\
\text { м/с }\end{array}$ & $\begin{array}{c}\text { Массовый расход, } \\
\text { кг/с }\end{array}$ & $\begin{array}{c}\text { Живое сечение, } \\
\text { м }^{2}\end{array}$ \\
\hline 2,75 & 12,3 & 2,4 & 114 & 0,0049 & $3,29 \mathrm{E}-05$ \\
\hline 2,75 & 12,0 & 2,34 & 113 & 0,0046 & $3,14 \mathrm{E}-05$ \\
\hline 2,75 & 11,7 & 2,28 & 111 & 0,0043 & $2,99 \mathrm{E}-05$ \\
\hline 1,25 & 12,3 & 2,4 & 181 & 0,0078 & $3,29 \mathrm{E}-05$ \\
\hline 1,25 & 12,0 & 2,34 & 178 & 0,0073 & $3,14 \mathrm{E}-05$ \\
\hline 1,25 & 11,7 & 2,28 & 175 & 0,0069 & $2,99 \mathrm{E}-05$ \\
\hline 0,25 & 12,3 & 2,4 & 480 & 0,0206 & $3,29 \mathrm{E}-05$ \\
\hline 0,25 & 12,0 & 2,34 & 471 & 0,0193 & $3,14 \mathrm{E}-05$ \\
\hline 0,25 & 11,7 & 2,28 & 463 & 0,0181 & $2,99 \mathrm{E}-05$ \\
\hline
\end{tabular}

Отличие результатов расчетов, приведенных в табл. 2 и 3 , состоит в том, что сначала проводились расчеты при одновременном уменьшении значений диаметра проволок и внутреннего диаметра трубы, а затем, наоборот, при уменьшении значений внутреннего диаметра трубы с одновременным увеличением значений диаметра проволок.

Во всех расчетах наблюдается явная линейная зависимость скорости течения кислорода и массового расхода от изменения значений площади живого сечения (площадь живого сечения включает в себя разность значений площади сечения трубы и проволок). 
Характеристики течения (при уменьшении значений внутреннего диаметра трубы с одновременным увеличением значения диаметра проволок)

\begin{tabular}{|c|c|c|c|c|c|}
\hline Длина, м & $\begin{array}{c}\text { Диаметр } \\
\text { трубы, мм }\end{array}$ & $\begin{array}{c}\text { Диаметр } \\
\text { проволок, мм }\end{array}$ & $\begin{array}{c}\text { Скорость, } \\
\text { м/с }\end{array}$ & $\begin{array}{c}\text { Массовый } \\
\text { расход, кг/с }\end{array}$ & $\begin{array}{c}\text { Живое сечение, } \\
\text { м }^{2}\end{array}$ \\
\hline 2,75 & 12,3 & 2,28 & 139 & 0,0075 & $4,12 \mathrm{E}-05$ \\
\hline 2,75 & 12,0 & 2,34 & 113 & 0,0046 & $3,14 \mathrm{E}-05$ \\
\hline 2,75 & 11,7 & 2,4 & 85 & 0,0024 & $2,16 \mathrm{E}-05$ \\
\hline 1,25 & 12,3 & 2,28 & 220 & 0,0119 & $4,12 \mathrm{E}-05$ \\
\hline 1,25 & 12,0 & 2,34 & 178 & 0,0073 & $3,14 \mathrm{E}-05$ \\
\hline 1,25 & 11,7 & 2,4 & 134 & 0,0038 & $2,16 \mathrm{E}-05$ \\
\hline 0,25 & 12,3 & 2,28 & 600 & 0,0324 & $4,12 \mathrm{E}-05$ \\
\hline 0,25 & 12,0 & 2,34 & 471 & 0,0193 & $3,14 \mathrm{E}-05$ \\
\hline 0,25 & 11,7 & 2,4 & 347 & 0,0098 & $2,16 \mathrm{E}-05$ \\
\hline
\end{tabular}

Для демонстрации линейной зависимости указанных характеристик проведен уточняющий расчет, результаты которого представлены на рис. 9.

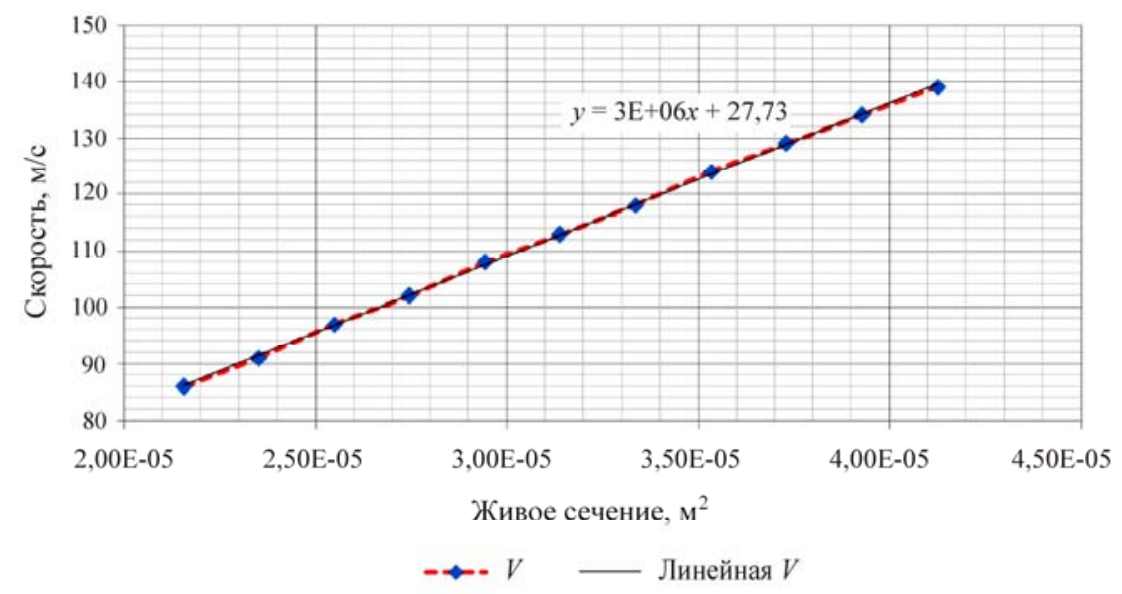

Рис. 9. Зависимость скорости от площади живого сечения

Из графика видно, что чем больше площадь живого сечения, тем больше и скорость истечения кислорода. При уменьшении площади живого сечения и, следовательно, величины каналов увеличивается гидравлическое сопротивление, что и вызывает падение скорости.

При попытке конструирования более мощного либо, наоборот, более экономичного кислородного копья становится необходимым разрешение задачи истечения кислорода для нестандартных диаметров труб. Одной из таких является труба диаметром $D_{\text {н }}=50$ мм и внутренним диаметром $d_{\text {внут }}=47$ мм, диаметр проволок остается равным 2,4 мм, варьирование значений длины находится также в пределах ранее заявленных трех значений. Исходные данные для кислорода прежние.

Результатом расчетов является, как и ожидалось, увеличение расхода и скорости истечения кислорода, что приведет к увеличению мощности копья (рис. 10, 11).

Таким образом, по сравнению с базовой серией расчетов на стандартном копье (см. табл. 1) наблюдается увеличение массового расхода в 20 раз при увеличении скорости в 1,235 раза. Столь сильное увеличение массового расхода объясняется серьезным увеличением площади живого сечения кислородного копья. Характер изменения скорости истечения 


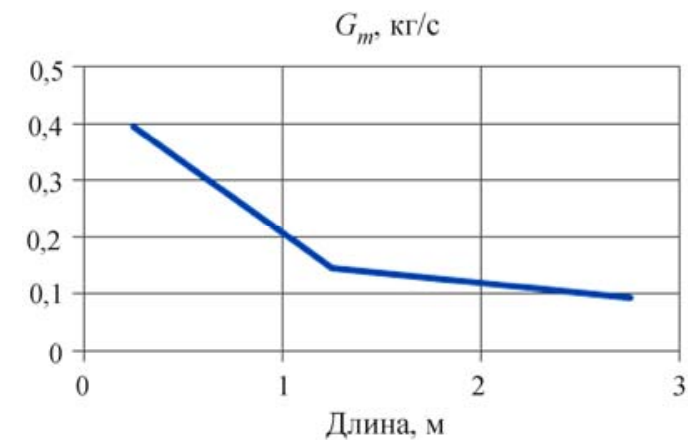

$a$

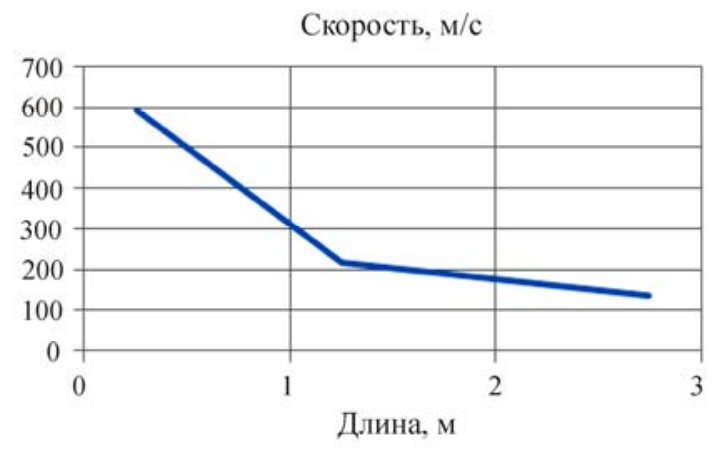

$\sigma$

Рис. 10. Результаты расчетов при конструировании более мощного или более экономичного копья:

$a$ - зависимость массового расхода от длины копья; $\sigma$ - зависимость скорости от длины копья

и расхода кислорода в зависимости от длины копья остался прежним. Стоит отметить, что число проволок, помещенных в копье, возрастет с 19 до 271 шт.

Следующим вариантом является изменение внутреннего диаметра кислородного копья до 6 мм и одновременное изменение диаметра проволок до 1,5 мм. При таких параметрах упаковка проволок в копье довольно рыхлая, что уменьшает гидравлическое сопротивление. Остальные параметры (плотность кислорода, кинематическая вязкость и т.п.) остаются без изменений.

Результаты расчета приведены на рис. 11.
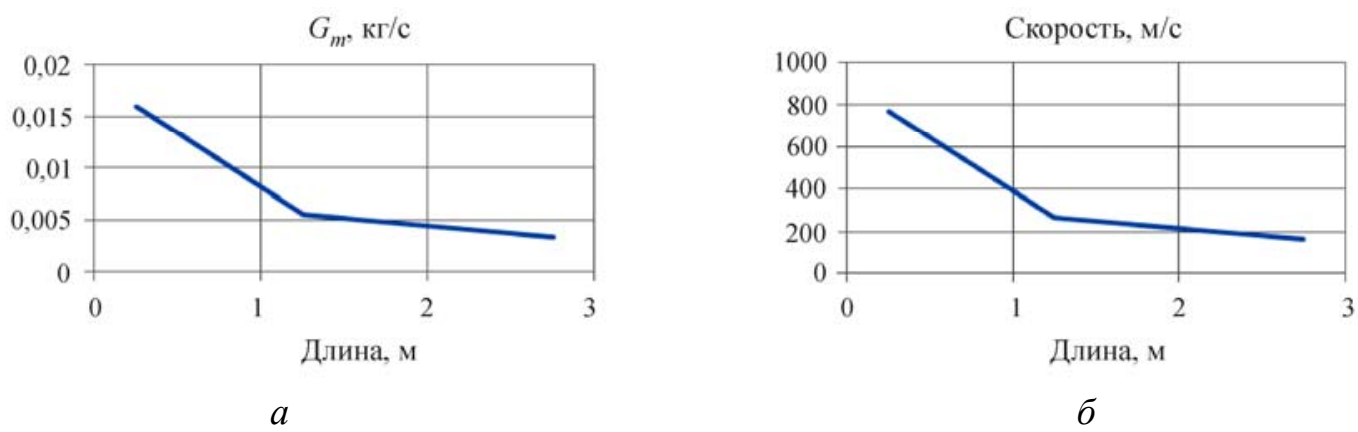

Рис. 11. Результаты расчетов копья с уменьшенным диаметром: $a$ - зависимость массового расхода от длины копья; $\sigma$ - зависимость скорости от длины копья

Результаты расчета по массовому расходу практически соответствуют результатам базового расчета (по максимальному значению: 0,016 против 0,0206 кг/с), в то время как скорости различаются практически в 2 раза. Можно прогнозировать некоторое уменьшение времени резки такими копьями за счет большой скорости истечения продуктов сгорания и, следовательно, выноса расплава из зоны реза. Как известно, расплав является дополнительным термическим сопротивлением и затрудняет процесс резки. Вынос расплава из зоны реза будет способствовать увеличению скорости резания ввиду постоянного взаимодействия пламени кислородного копья с поверхностью разрезаемого материала.

Еще одной характеристикой, требующей варьирования, является избыточное давление на входе в кислородное копье [15]. Интерес представляет изменение давления как в бо́льшую, так и в меньшую сторону, при этом фиксированное избыточное давление равняется 12 бар.

Для этого была проведена серия расчетов с избыточным давлением 8 и 16 бар на стандартном, увеличенном и уменьшенном копьях стандартной длины в 2,75 м (без мундштука). Будем полагать, что вариант 1 есть стандартное кислородное копье, вариант 2 - копье с увеличенным внутренним диаметром до 47 мм, вариант 3 - уменьшенное кислородное копье.

Результаты расчета сведены в табл. 4, а скорости представлены на рис. 12. 
Результаты расчетов с измененным давлением

\begin{tabular}{|c|c|c|c|c|c|c|}
\hline \multirow{2}{*}{$P$, бар } & \multicolumn{2}{|c|}{ Вариант 1 } & \multicolumn{2}{c|}{ Вариант 2 } & \multicolumn{2}{c|}{ Вариант 3 } \\
\cline { 2 - 7 } & $G_{m}$, кг/c & $V, \mathrm{~m} / \mathrm{c}$ & $G_{m}$, кг/c & $V, \mathrm{~m} / \mathrm{c}$ & $G_{m}$, кг/c & $V, \mathrm{M} / \mathrm{c}$ \\
\hline 8 & 0,0039 & 91 & 0,0726 & 109 & 0,0028 & 133 \\
\hline 12 & 0,0049 & 114 & 0,0919 & 138 & 0,0035 & 168 \\
\hline 16 & 0,0058 & 135 & 0,1079 & 162 & 0,0041 & 198 \\
\hline
\end{tabular}

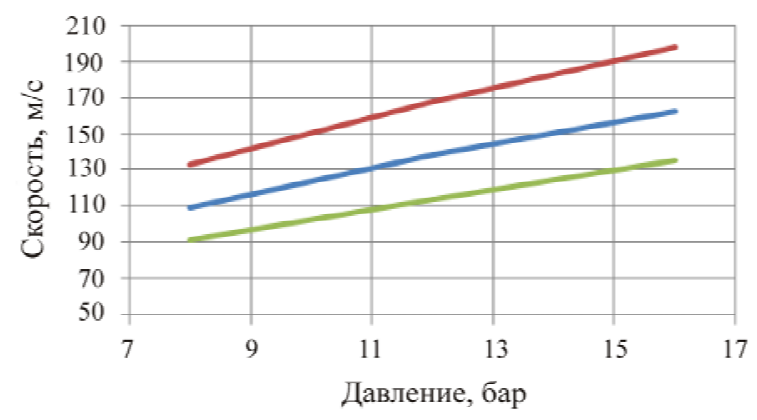

Рис. 12. Распределение скоростей: — вариант 1 ; — вариант 2 ; — вариант 3

Распределение значений скорости в зависимости от роста давления практически линейно, но при более детальном анализе (рис. 13) проявляется явная нелинейность. На рис. 13 демонстрируется изменение скорости с ростом давления для 2-го варианта.

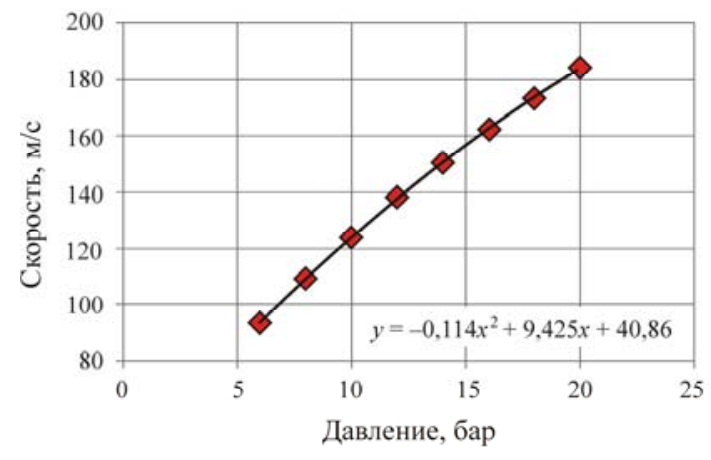

Рис. 13. Распределение значений скорости при увеличенном внутреннем диаметре до 47 мм (вариант 2): $\diamond$ скорость, м/с; — тренд

Учитывая скоростные и расходные характеристики, полученные на этом этапе расчетов, можно переходить к непосредственному моделированию процесса резки материалов кислородным копьем.

\section{Выводы}

1. Решена задача горения двух различных материалов в кислороде: алюминия, железа, а также их смеси, с учетом различного соотношения горючего и окислителя.

2. Получены результаты расчетно-теоретического исследования процесса горения кислородного копья и его взаимодействия с различными материалами.

3. Представленные результаты дают основание для проведения дальнейшей работы по совершенствованию методики проектирования кислородного копья, обеспечивающей требуемое качество управления технологическим процессом разделительной резки крупногабаритных и толстостенных металлических и неметаллических конструкций. 


\section{Библиографический список}

1. Сухунин Г.К., Трофимов А.А. Ручная кислородная резка. - М.: Машиностроитель, 1974. - 88 с.

2. Спектор О.Ш., Осиновская Г.А. Кислородная резка в металлургии. - М.: Металлург, 1972. - 168 с.

3. Рыбаков В.М. Сварка и резка металлов. - М.: Высшая школа, 1979. - 214 с.

4. Банов М.Д., Казаков Ю.В. Сварка и резка металлов. - М.: Академия, 2002. - 399 с.

5. Крюков А.Ю., Малинин В.И. Математическое моделирование горения переобогащенной алюминиево-воздушной смеси на основе неравновесной термодинамики процессов // Физика горения и взрыва. - 2018. - Т. 54, № 1. - С. 39-51.

6. Branderberger E. New oxygen wire-core lances // Metal construction. - 1975. - Vol. 7. - 623 p.

7. Slottman G.V., Roper E.H. Oxygen cutting. - New York: McGraw-Hill, 1951. - P. 143-145.

8. Grosse A.V., Conway J.B. Combustion of metals in oxygen // Ind. Eng. Chem. - 1958. - No. 50. P. 4663-4669.

9. Symposium on flammability and sensitivity of materials in oxygen-enriched atmospheres / S. Sircar, H. Gable, J. Stoltzfus, F. Benz; eds. J.M. Stoltzfus, K. McCilroy. - Philadelphia, Pa.: ASTM, 1991. - Vol. 5. P. 123-131.

10. Sato J., Hirano T. Symposium on flammability and sensitivity of materials in oxygen-enriched atmospheres / ed. M.A. Benning. - Philadelphia. Pa.: ASTM, 1986. - P. 118-134.

11. Земерев Е.С., Малинин В.И. Неравновесное изотермическое критическое истечение порошковогазовой среды из отверстия // Вестник Казан. технол. ун-та. - 2017. - Т. 20, № 1. - С. 57-61.

12. Земерев Е.С., Малинин В.И. Анализ течения порошковогазовой среды в коническом канале с выпускным отверстием // Вестник Пермского национального исследовательского политехнического университета. Аэрокосмическая техника. - 2016. - № 47. - С. 154-176.

13. Крюков А.Ю., Малинин В.И. Расчет содержания субоксидов в продуктах сгорания алюминиевовоздушной смеси по модели неравновесной термодинамики // Вестник Пермского национального исследовательского политехнического университета. Аэрокосмическая техника. - 2016. - № 44. - С. 116-131.

14. Крюков А.Ю., Малинин В.И. Расчет горения полифракционной аэровзвеси частиц алюминия по модели неравновесной термодинамики // Вестник Казан. технол. ун-та. - 2016. - Т. 19, № 12. - С. $128-131$.

15. Hirano T., Sato K., Sato Y. Prediction of metal fire spread in high pressure oxygen // Combustion Sci. and Technol. - 1983. - No. 32. - P. 137-159.

\section{References}

1. Sukhunin G.K. Trofimov A.A. Ruchnaya kislorodnaia rezka [Manual oxygen cutting]. Moscow: Mashinostroitel, 1974, $88 \mathrm{p}$.

2. Spector O.S., Osinovskaia G.A. Kislorodnaia rezka v metallurgii [Oxygen cutting in metallurgy]. Moscow: Metallurg, 1972, 168 p.

3. Rybakov V.M. Svarka I rezka metallov [Welding and cutting of metals]. Moscow: Vysshaia shkola, 1979,214 p.

4. Banov M.D., Kazakov Y.V. Svarka I rezka metallov [Welding and cutting of metals]. Moscow: Akademiya, 2002, 399 p.

5. Kryukov A.Yu., Malinin V.I. Matematicheskoye modelirovaniye goreniya pereobogashchonnoy alyuminiyevo-vozdushnoy smesi na osnove neravnovesnoy termodinamiki protsessov [Computation of burn-out of polyfractional aluminum air-suspension with taking into account of suboxide alo interaction with particles surface]. Combustion, explosion and shock waves, 2018, vol. 54, no. 1, pp. 39-51.

6. Branderberger E. New oxygen wire-core lances, Metal construction, 1975, vol. 7, 623 p.

7. Slottman G.V., Roper E.H. Oxygen Cutting. New York: McGraw-Hill, 1951, pp. 143-145.

8. Grosse A.V., Conway J.B. Combustion of metals in oxygen. Ind. Eng. Chem. 1958, no. 50, pp. 4663-4669.

9. Sircar S., Gable H., Stoltzfus J., Benz F. Symposium on Flammability and Sensitivity of Materials in Oxygen-Enriched Atmospheres: Fifth Volume. Eds. J. M. Stoltzfus and K. McCilroy. Philadelphia, Pa.: ASTM, 1991, pp. 123-131.

10. Sato J., Hirano T. Symposium on Flammability and Sensitivity of Materials in Oxygen-Enriched Atmospheres: Second Volume. Ed. M. A. Benning. Philadelphia. Pa.: ASTM, 1986, vol. 5, pp. 118-134.

11. Zemerev E.S., Malinin V.I. Neravnovesnoye izotermicheskoye kriticheskoye istecheniye poroshkovogazovoy sredy iz otverstiya [Nonequilibrium isothermal critical outflow of a powder-gas medium from an opening]. KNRTU Bulletin, 2017, vol. 20, no. 1, pp. 57-61. 
12. Zemerev E.S., Malinin V.I. Analiz techeniya poroshkovogazovoy sredy v konicheskom kanale s vypusknym otverstiyem [Analysis of the powder-gas flow in a conical channel with an outlet hole]. PNRPU Aerospace Engineering Bulletin, 2016, no. 47, pp. 154-176.

13. Kryukov A.Yu., Malinin V.I. Raschot soderzhaniya suboksidov v produktakh sgoraniya alyuminiyevovozdushnoy smesi po modeli neravnovesnoy termodinamiki [Computation of suboxides in the combustion products of aluminium air mixture by model of nonequilibrium thermodynamics]. PNRPU Aerospace Engineering Bulletin, 2016, no. 44, pp. 116-131.

14. Kryukov A.Yu., Malinin V.I. Raschot goreniya polifraktsionnoy aerovzvesi chastits alyuminiya po modeli neravnovesnoy termodinamiki [Calculation of combustion of multifractional aerosuspension of aluminum particles according to the model of nonequilibrium thermodynamics]. KNRTU Bulletin, 2016, vol. 19, no. 12 , pp. $128-131$.

15. Hirano T., Sato K., Sato Y. Prediction of metal fire spread in high pressure oxygen. Combustion Science and Technology, 1983, no. 32, pp. 137-159.

\section{Об авторах}

Цветков Юрий Викторович (Пермь, Россия) - директор ООО МИП «Энергомашиностроение» (614010, г. Пермь, ул. Героев Хасана, д. 12-7, e-mail: cuv-vt@ perm.ru).

Цветков Геннадий Александрович (Пермь, Россия) - доктор технических наук, профессор кафедры «Геофизика» ФГБОУ ВО ПГНИУ; кафедры «Безопасность жизнедеятельности» ФГБОУ ВО ПНИПУ (614990, г. Пермь, Комсомольский пр., д. 29, e-mail: zvetkov71043@ mail.ru).

Цветков Роман Валерьевич (Пермь, Россия) - кандидат технических наук, научный сотрудник лаборатории интеллектуального мониторинга ИМСС УрО РАН (614013, г. Пермь, ул. Королева, д. 1, e-mail: flower@icmm.ru).

Малинин Владимир Игнатьевич (Пермь, Россия) - доктор технических наук, профессор кафедры «Ракетно-космическая техника и энергетические системы» ФГБОУ ВО ПНИПУ (614990, г. Пермь, ул. Комсомольский пр., д. 29, e-mail: malininvi@ mail.ru).

Савин Максим Анатольевич (Пермь, Россия) - кандидат технических наук, доцент кафедры «Сварочное производство, метрология и технология материалов» ФГБОУ ВО ПНИПУ (614990, г. Пермь, Комсомольский пр., д. 29, e-mail: abins@pstu.ru).

Жижилев Николай Евгеньевич (Пермь, Россия) - начальник испытательного цеха ПАО «Протон - Пермские моторы» (614010, г. Пермь, Комсомольский пр., д. 93, e-mail: zhizhileff.nikolai@yandex.ru).

\section{About the authors}

Yuriy V. Tsvetkov (Perm, Russian Federation) - Director of the Small Innovative Enterprise "Energomashinostroenie" (12-7, Geroyev Khasana st., Perm, 614010, Russian Federation, e-mail: cuv-vt@perm.ru).

Gennadiy A. Tsvetkov (Perm, Russian Federation) - Doctor of Technical Sciences, Professor of Geophysics Department, Perm State National Research University; of Life Safety Department, Perm National Research Polytechnic University (29, Komsomolsky av., Perm, 614990, Russian Federation, e-mail: zvetkov71043@mail.ru).

Roman V. Tsvetkov (Perm, Russian Federation) - CSc in Technical Sciences, Researcher of Intellectual Monitoring Laboratory, Institute of Continuous Media Mechanics of the Ural Branch of the Russian Academy of Science (1, Koroleva st., Perm, 614013, Russian Federation, e-mail: flower@icmm.ru).

Vladimir I. Malinin (Perm, Russian Federation) - Doctor of Technical Sciences, Professor of Rocket and Space Engineering Systems Department, Perm National Research Polytechnic University (29, Komsomolsky av., Perm, 614990, Russian Federation, e-mail: malininvi@ mail.ru).

Maksim A. Savin (Perm, Russian Federation) - CSc in Technical Sciences, Associate Professor of Welding Production Department, Metrology and Technology of Materials Department, Perm National Research Polytechnic University (29, Komsomolsky av., Perm, 614990, Russian Federation, e-mail: abins@ pstu.ru).

Nikolay E. Zhizhilev (Perm, Russian Federation) - Head of the Test Shop Public Joint Stock Company "Proton - Permskiye motory" (93, Komsomolsky av., Perm, 614010, Russian Federation, e-mail: zhizhileff.nikolai@yandex.ru). 